\title{
The role of periampullary diverticulum on the incidence of pancreaticobiliary diseases and the outcome of endoscopic retrograde cholangiopancreatography
}

Qiaofeng Chen, Yuanyuan Zhang, Zhihua Tang, Mingju Yu, Zhijian Liu, Xiaojiang Zhou, Guohua Li, Youxiang Chen, Xiaodong Zhou

The First Affiliated Hospital of Nanchang University, Nanchang, China

Submitted: 3 March 2018; Accepted: 19 August 2018

Online publication: 22 April 2020

Arch Med Sci 2021; 17 (4): 905-914

DOI: https://doi.org/10.5114/aoms.2020.94591

Copyright $\odot 2020$ Termedia \& Banach

\section{Abstract}

Introduction: Periampullary diverticulum (PAD), although commonly discovered in patients undergoing endoscopic retrograde cholangiopancreatography $(E R C P)$, remains controversial regarding its role in pancreaticobiliary diseases and the failure rate of cannulation. The aim of this study was to evaluate the association of PAD with pancreaticobiliary diseases and its impact on the outcome of ERCP.

Material and methods: A retrospective analysis was carried out on $1455 \mathrm{pa}$ tients who underwent an ERCP. Patients were divided into a PAD group and a control group without PAD, and propensity score matching was performed to adjust for clinical differences. The comparison was focused on pancreaticobiliary diseases, technical success, and complications of ERCP.

Results: The occurrence of PAD is associated significantly with increasing age $(p<0.001)$. Incidences of acute pancreatitis (AP), suppurative cholangitis, and pancreatic head cancer were significantly higher in the PAD group $(p<0.05)$. After propensity score matching, the PAD group exhibited a higher rate of post-ERCP complications including haemorrhage, post-ERCP pancreatitis (PEP), and hyperamylasaemia $(p<0.05)$. However, the prevalence of perforation and the success rate of ERCP did not differ between groups $(p>0.05)$.

Conclusions: Periampullary diverticulum develops with aging and seems to be associated with an increase in pancreaticobiliary diseases and post-ERCP complications except for perforation. Additionally, the presence of PAD does not affect the technical success of ERCP.

Key words: biliary calculi, complications, common bile duct, endoscopic retrograde cholangiopancreatography, periampullary diverticulum.

\section{Introduction}

Periampullary diverticulum (PAD) refers to a mucosal or submucosal outpouching of duodenum commonly caused by a defect of the local muscular layer and arising within a $2 \sim 3 \mathrm{~cm}$ radius of the major papilla [1, 2]. The duodenum, second to the colon, is the most frequent site for gastrointestinal diverticulum [3]. Most of these herniations are composed of acquired cystic lesions [4], with a higher prevalence in individuals 50 to 60 years old. Periampullary diverticulum is usually asymptomatic [5], and only $5 \%$ of patients develop complications such as bleeding,
Corresponding author: Xiaodong Zhou

Department of Gastroenterology The First Affiliated Hospital of Nanchang University 17 Yongwaizheng St Nanchang 330006, China Phone: +8613767038587 E-mail: yfyzhouxd@163.com 
perforation, and pancreatitis. Generally, the association of PAD with pancreatitis, biliary obstruction, choledocholithiasis, and rarely cancer has been called Lemmel's syndrome or juxtapapillary syndrome [6]. The relatively high prevalence of PAD (around 5-23\%) documented in previous studies [7] underscores the significant influence of these luminal defects on pancreaticobiliary diseases or the procedure of endoscopic retrograde cholangiopancreatography (ERCP). Whether PAD makes the ERCP procedure technically challenging and increases the risks of postoperative complications or has a significant impact on the prevalence of those specific conditions has not yet been firmly established and will constitute the focus of our study.

\section{Material and methods}

\section{Patients}

Patients who underwent therapeutic ERCP in an inpatient setting from January 2016 to December 2016 were eligible for enrolment. ERCP was performed on cases when imaging or laboratory examinations indicated that therapeutic management was necessary or the diagnosis was unclear. Patients younger than 18 years old were excluded. This study was approved by the Ethical Committee of the First Affiliated Hospital of Nanchang University. Due to its retrospective nature, written informed consent could not be obtained from all patients.

\section{Methods and procedures}

All medical records including patient charts, laboratory findings (blood routine, coagulation function, liver and renal functions, hemodlastase, etc.), and various imaging studies (magnetic resonance cholangiopancreatography (MRCP), abdominal ultrasonography, and computed tomography (CT)) were reviewed to evaluate each patient's general condition prior to the operation. All ERCP procedures were performed by highly experienced pancreatobiliary endoscopists ( $>2000$ procedures performed) using a standard therapeutic duodenoscopy. Clinical parameters, including papilla's location, shape, or opening, PAD type, diameter, and number were recorded during duodenoscopy. After wire-guided and selective bile duct or pancreatic duct cannulation, cholangiography was performed with a standard iodinated contrast medium to verify the existence of cholangiopancreatic lesions. Subsequent therapeutic manoeuvres were performed according to the situation. For instance, in patients with common bile duct (CBD) stones, endoscopic sphincterotomy (EST), endoscopic papillary balloon dilation (EPBD), or a combination of both was chosen for stone extraction. Finally, basket exploration or nasal cholangiography was carried out to confirm whether the stones had been completely removed. Patients with non-removable large stones, biliary tract stricture, or suppurative infection were subjected to either nasobiliary drainage or placement of a biliary stent. Treatment efficacy represented by improvement of liver function, successful stone extraction, and incidence of ERCP-related complications was monitored and recorded.

\section{Classification of periampullary diverticulum and definition of giant diverticulum}

From the endoscopic view, PAD is classified as type I, II, or III according to its location relative to the major papilla $[1,8,9]$. In type I the papilla is located inside the diverticulum (Figure $1 \mathrm{~A}$ ), while in type II and III the papilla is located, respectively, at the edge and the outside (commonly within $2 \mathrm{~cm}$ of the diverticulum) of the diverticulum (Figures $1 \mathrm{~B}, \mathrm{C}$ ). Diverticulum with a diameter $>3 \mathrm{~cm}$ is referred to as "giant diverticulum" [10].

\section{Definitions of post-endoscopic retrograde cholangiopancreatography complications}

PEP refers to the presence of new, or the worsening of existing, abdominal pain with a threefold elevation of serum amylase, all within $24 \mathrm{~h}$ post-ERCP [11]. Bleeding is considered when a transfusion, or angiographic or surgical intervention is required and is graded as mild (clinical (not merely endoscopic) evidence of bleeding; haemoglobin drop $<3 \mathrm{~g} / \mathrm{dl}$ and no necessity of transfusion), moderate (transfusion $\leq 4$ units, no surgery or angiographic intervention) and severe (transfusion ( $\geq 5$ units) or intervention (surgical or angiographic)) [11] (Figure 2). Perforation is defined by the presence of contrast agent or air in the retroperitoneal space (Figure 3). Hyperamylasaemia is determined by a serum amylase level four or five times higher than the normal upper limit within 2-24 $\mathrm{h}$ post-ERCP, all without the simultaneous presence of abdominal pain, nausea, or vomiting [12].

\section{Statistical analysis}

In order to adjust for the influences of confounding factors and investigate the actual role of PAD on the technical success and complications of ERCP, patients of the non-PAD group were matched to those in the PAD group with one-toone propensity score matching. Age, gender, and concomitant diseases were used as matching factors in this study. The propensity score was calculated using a logistic regression analysis model. Because there was no major confounder affecting the incidence of pancreaticobiliary diseases between the two groups, simple comparative analysis was performed. 

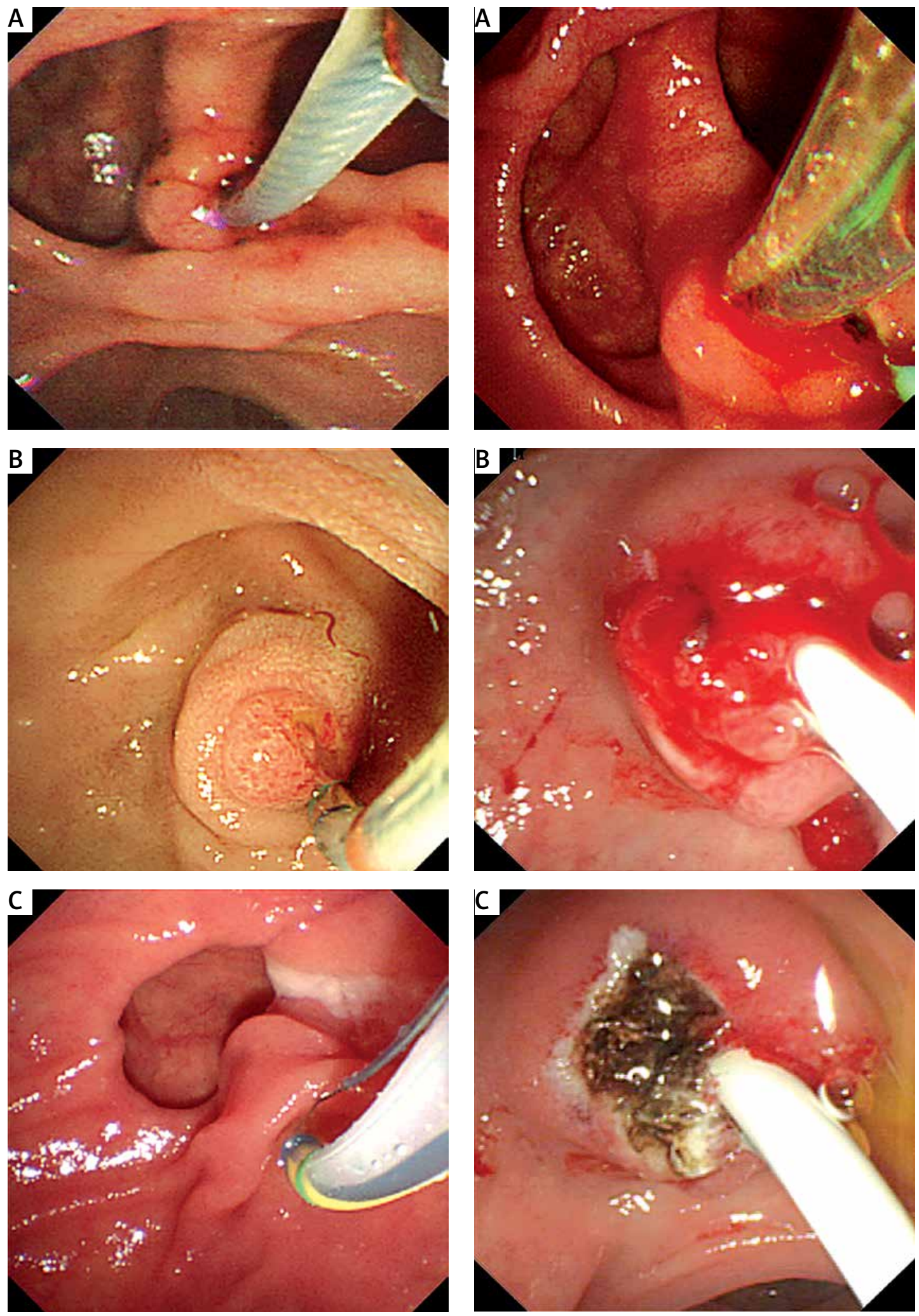

Figure 1. Endoscopic view of a papilla located inside the diverticulum (type I, A), in the margin of the diverticulum (type II, B), and outside of the diverticulum (type III, C)

Figure 2. Intraoperative haemorrhage in a patient with periampullary diverticulum (PAD) (A) and without PAD (B) during endoscopic retrograde cholangiopancreatography. Haemorrhage was coagulated by argon plasma coagulation (C) 

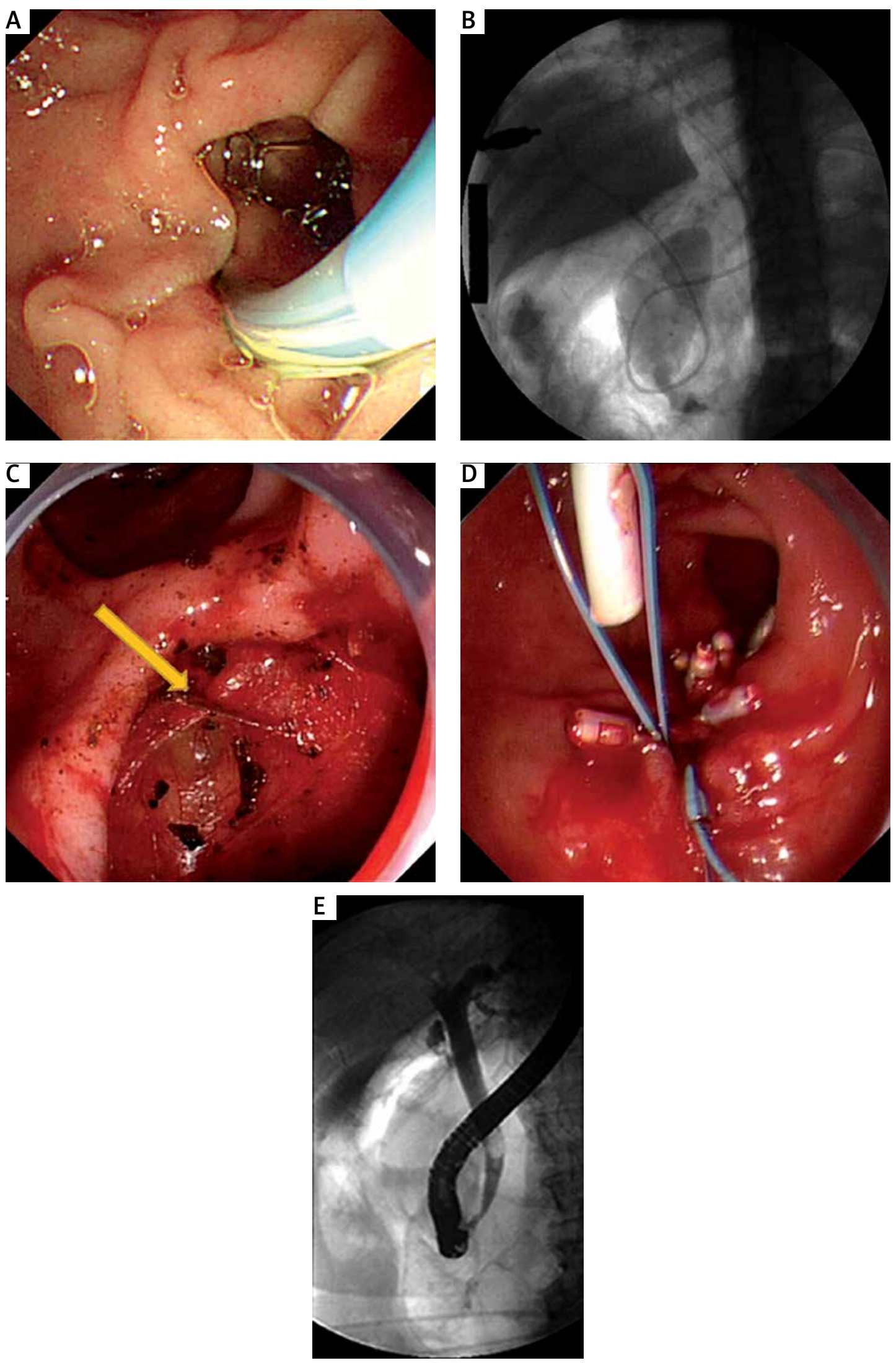

Figure 3. Intraoperative perforation in a patient with periampullary diverticulum (PAD) (A) and without PAD (arrow, C) during endoscopic retrograde cholangiopancreatography. X-ray showing substantial pneumatosis around the right kidney in a patient with PAD (B) and without PAD (E). Closure of the perforation by suturing the pouch with a nylon loop and titanium clips (D) 
Table I. Baseline clinical characteristics and comparison of pancreaticobiliary diseases

\begin{tabular}{|c|c|c|c|c|}
\hline \multirow[t]{2}{*}{ Parameter } & \multicolumn{3}{|c|}{ Group, $n(\%)$} & \multirow[t]{2}{*}{$P$-value } \\
\hline & Total & $\operatorname{PAD}(n=293)$ & Non-PAD $(n=1162)$ & \\
\hline \multicolumn{5}{|l|}{ Sex: } \\
\hline Male & 728 & $152(20.88)$ & $576(79.12)$ & \multirow[t]{2}{*}{0.480} \\
\hline Female & 727 & $141(19.39)$ & $586(80.61)$ & \\
\hline \multicolumn{2}{|l|}{ Age (mean \pm SD) [years]: } & $66 \pm 15.72$ & $54.0 \pm 17.05$ & \\
\hline$<40$ & 348 & $13(3.74)$ & $335(96.26)$ & \multirow[t]{5}{*}{$<0.001^{*}$} \\
\hline $40-49$ & 483 & $65(13.46)$ & $418(86.54)$ & \\
\hline $50-59$ & 139 & $39(28.06)$ & $100(71.94)$ & \\
\hline $60-69$ & 163 & $55(33.74)$ & $108(66.26)$ & \\
\hline$>70$ & 322 & $121(37.58)$ & $201(62.42)$ & \\
\hline \multicolumn{3}{|l|}{ CBD stone diameter $[\mathrm{cm}]:$} & & 0.052 \\
\hline$<1$ & 863 & $200(83.33)$ & $663(76.12)$ & $0.017^{*}$ \\
\hline $1 \sim 2$ & 191 & $28(11.67)$ & $163(18.71)$ & $0.010^{\star}$ \\
\hline $2 \sim 3$ & 40 & $7(2.92)$ & $33(3.79)$ & 0.521 \\
\hline$>3$ & 17 & $5(2.08)$ & $12(1.38)$ & 0.430 \\
\hline \multicolumn{5}{|l|}{ CBD stone number: } \\
\hline Multiple & 662 & $164(68.33)$ & $498(57.18)$ & \multirow[t]{2}{*}{$0.002^{*}$} \\
\hline Solitary & 449 & $76(31.67)$ & $373(42.88)$ & \\
\hline Biliary calculi: & 1111 & $240(81.91)$ & $871(74.96)$ & $<0.001^{*}$ \\
\hline Cholecystolithiasis with CBD stones & 383 & $58(19.80)$ & $325(27.97)$ & $0.005^{\star}$ \\
\hline Primary CBD stones & 412 & $112(38.23)$ & $300(25.82)$ & $<0.001^{*}$ \\
\hline CBD stones after cholecystectomy & 316 & $70(23.89)$ & $246(21.17)$ & 0.313 \\
\hline AP & 158 & $66(22.53)$ & $92(7.92)$ & $<0.001^{*}$ \\
\hline Suppurative cholangitis & 183 & $56(19.11)$ & $127(10.93)$ & $<0.001^{*}$ \\
\hline Cholangiocarcinoma & 145 & $25(8.53)$ & $120(10.33)$ & 0.359 \\
\hline Papillary carcinoma of duodenum & 18 & $2(0.68)$ & $16(1.38)$ & 0.506 \\
\hline Duodenal adenomas & 11 & $1(0.34)$ & $10(0.86)$ & 0.589 \\
\hline Pancreatic head cancer & 41 & $14(4.78)$ & $27(2.32)$ & $0.023^{*}$ \\
\hline Biliary stent removal & 92 & $4(1.37)$ & $88(7.57)$ & $<0.001^{*}$ \\
\hline Chronic pancreatitis & 14 & $3(1.02)$ & $11(0.95)$ & 1.000 \\
\hline Biliary ascariasis & 2 & $1(0.34)$ & $1(0.09)$ & 0.362 \\
\hline Unknown aetiology of CBD dilatation & 21 & $3(1.02)$ & $18(1.55)$ & 0.690 \\
\hline
\end{tabular}

$P A D$ - periampullary diverticulum, $A P$ - acute pancreatitis, $C B D$ - common bile duct. *Statistically significant.

All statistical analyses were performed using SPSS version 19.0. Categorical variables were displayed as numbers (percentages) and analysed with the $\chi^{2}$ test and Fisher's exact test as appropriate. Continuous variables were expressed as mean \pm standard deviation (SD) and compared us- ing Student's $t$-test. $P$-values less than 0.05 were considered statistically significant.

\section{Results}

A total of 1455 patients who underwent ERCP during the period January 2016 to December 2016 
were included. Of these, 293 patients were identified as having PAD (20.14\%), including 152 males and 141 females. The remaining 1162 comprised 576 males and 586 females. The comparison of PAD frequency between the two groups showed a significantly higher mean age in the PAD group $(66.0 \pm 15.72$ vs. $54.0 \pm 17.05$ years; $p<0.001)$ and indicated that the incidence of PAD increases with age, whereas no difference in the male-to-female ratio was observed $(p>0.05)$ (Table $\mathrm{I}$ ).

Additionally, the diameter of CBD stones varied between the PAD and non-PAD groups. A statistically significant difference can be observed between the two groups at $D \leq 2 \mathrm{~cm}(p<0.05)$. Moreover, a significant difference in the number of CBD stones between the two groups and a higher occurrence of multiple CBD stones in the PAD group were observed $(p=0.002$ ) (Table I).

The PAD group showed significantly higher incidences of primary CBD stones, suppurative cholangitis, and AP compared to the control group $(p<0.05)$. Nevertheless, the occurrence rate of a combination of cholecystolithiasis and CBD stones as well as the risk of biliary stent removal were found to be much higher in the control group $(p<0.05)$ (Table I). Of the 1455 patients, $158(10.86 \%)$ cases of patients with AP were discovered pre-ERCP (66 with PAD, 92 without
PAD). These patients were categorised according to the aetiology of AP. Seventy-three of those cases were detected in cholecystolithiasis with CBD stone patients $(39.66 \%$ in PAD vs. $15.38 \%$ in non-PAD), 112 cases were reported in primary CBD stone patients ( $20.54 \%$ vs. $8.67 \%), 70$ cases in CBD stone after cholecystectomy patients (15.71\% vs. $3.66 \%), 25$ cases in cholangiocarcinoma patients ( $16.00 \%$ vs. $2.50 \%)$, and 14 cases in patients with pancreatic head cancer $(28.57 \%$ vs. $3.70 \%)$. All differences were significant $(p<0.05)$ (Table II).

Furthermore, the result indicated a correlation between PAD type and the incidence of suppurative cholangitis and giant diverticulum ( $p<0.05)$. However, no association was observed in cases of AP, PEP, and hyperamylasaemia $(p>0.05)$. Interestingly, suppurative cholangitis incidence in PAD type III and that of giant diverticulum in PAD type I were significantly higher than in the remaining subgroups $(p<0.015)$ (Table III).

Complications of ERCP were compared between the two groups (Table IV). Gastrointestinal bleeding during the procedure was observed in $7.17 \%$ and $3.96 \%$ of those patients in the PAD and non-PAD groups, respectively. This indicated that bleeding was evidently more frequent in patients with PAD $(p=0.019)$. However, in terms

Table II. Comparison of pancreaticobiliary diseases and complications in different periampullary diverticulum subtypes $(n(\%))$

\begin{tabular}{|lcccc|}
\hline Disease & Type I $(n=56)$ & Type II $(n=85)$ & Type III $(n=152)$ & $P$-value \\
\hline Biliary calculi & $44(78.57)$ & $67(78.82)$ & $129(84.87)$ & 0.393 \\
\hline Suppurative cholangitis & $5(8.93)$ & $10(11.76)$ & $41(26.97)$ & $0.002^{*}$ \\
\hline AP & $10(17.86)$ & $20(23.53)$ & $36(23.68)$ & 0.649 \\
\hline Giant diverticulum & $12(21.43)$ & $6(7.06)$ & $13(8.55)$ & $0.013^{*}$ \\
\hline PEP & $5(8.93)$ & $8(9.41)$ & $11(7.24)$ & 0.822 \\
\hline Hyperamylasaemia & $10(17.86)$ & $10(11.76)$ & $15(9.87)$ & 0.288 \\
\hline
\end{tabular}

$A P$ - acute pancreatitis, PEP - post-endoscopic retrograde cholangiopancreatography pancreatitis. *Statistically significant.

Table III. Comparison of the incidence of AP between periampullary diverticulum and non-periampullary diverticulum groups

\begin{tabular}{|c|c|c|c|}
\hline \multirow[t]{2}{*}{ Aetiologies of AP } & \multicolumn{2}{|c|}{ Group, $n(\%)$} & \multirow[t]{2}{*}{$P$-value } \\
\hline & PAD & Non-PAD & \\
\hline Cholecystolithiasis with CBD stones & $23(39.66)$ & $50(15.38)$ & $<0.001^{*}$ \\
\hline Primary CBD stones & $23(20.54)$ & $29(8.67)$ & $0.003^{*}$ \\
\hline CBD stones after cholecystectomy & $11(15.71)$ & $9(3.66)$ & $<0.001^{\star}$ \\
\hline Cholangiocarcinoma & $4(16.00)$ & $3(2.50)$ & $0.017^{\star}$ \\
\hline Pancreatic head cancer & $4(28.57)$ & $1(3.70)$ & $0.039^{*}$ \\
\hline Unknown aetiology & $1(33.33)$ & $0(0)$ & 0.143 \\
\hline
\end{tabular}

$A P$ - acute pancreatitis, $P A D$ - periampullary diverticulum, $C B D$ - common bile duct. *Statistically significant. 
Table IV. Comparison of technical success and complications of endoscopic retrograde cholangiopancreatography before and after propensity score matching

\begin{tabular}{|lcccccccc|}
\hline Parameter & \multicolumn{3}{c}{ Before PS matching } & \multicolumn{5}{c|}{ After PS matching } \\
\cline { 2 - 9 } & Total & $\begin{array}{c}\text { PAD } \\
(n=293)\end{array}$ & $\begin{array}{c}\text { Non-PAD } \\
(n=1162)\end{array}$ & $P$-value & Total & $\begin{array}{c}\text { PAD } \\
(n=206)\end{array}$ & $\begin{array}{c}\text { Non-PAD } \\
(n=206)\end{array}$ & $P$-value \\
\hline Failed cannulation & 15 & $3(1.02)$ & $12(1.03)$ & 1.000 & 8 & $3(1.45)$ & $5(2.43)$ & 0.721 \\
\hline \begin{tabular}{l} 
Complications of ERCP: \\
\hline Haemorrhage:
\end{tabular} & 67 & $21(7.17)$ & $46(3.96)$ & $0.019^{*}$ & 26 & $17(8.25)$ & $7(3.40)$ & $0.035^{*}$ \\
\hline Mild & 59 & $18(6.14)$ & $41(3.53)$ & $0.043^{*}$ & 22 & $14(6.80)$ & $6(2.91)$ & 0.067 \\
\hline Moderate & 6 & $2(0.68)$ & $4(0.34)$ & 0.348 & 3 & $2(0.97)$ & $1(0.49)$ & 1.000 \\
\hline Severe & 2 & $1(0.34)$ & $1(0.09)$ & 0.362 & 1 & $1(0.49)$ & $0(0)$ & 1.000 \\
\hline $\begin{array}{l}\text { Perforation } \\
\text { PEP }\end{array}$ & 4 & $3(1.02)$ & $1(0.09)$ & $0.028^{*}$ & 2 & $2(1.00)$ & $0(0)$ & 0.499 \\
\hline Hyperamylasaemia & 116 & $35(11.95)$ & $81(6.97)$ & $0.005^{*}$ & 44 & $29(14.08)$ & $15(7.28)$ & $0.026^{*}$ \\
\hline
\end{tabular}

$P S$ - propensity score, $P A D$ - periampullary diverticulum, ERCP - endoscopic retrograde cholangiopancreatography, PEP - post-ERCP pancreatitis. *Statistically significant.

Table V. Clinical characteristics according to periampullary diverticulum status after propensity score matching

\begin{tabular}{|c|c|c|c|c|}
\hline \multirow[t]{2}{*}{ Parameter } & \multicolumn{3}{|c|}{ Group, $n(\%)$} & \multirow[t]{2}{*}{$P$-value } \\
\hline & Total & $\operatorname{PAD}(n=206)$ & Non-PAD $(n=206)$ & \\
\hline \multicolumn{5}{|l|}{ Sex: } \\
\hline Male & 246 & $123(59.71)$ & $123(59.71)$ & \multirow{2}{*}{1.00} \\
\hline Female & 166 & $83(40.29)$ & $83(40.29)$ & \\
\hline Age (mean \pm SD) [years]: & & $62.79 \pm 15.84$ & $59.87 \pm 18.17$ & 0.083 \\
\hline Biliary calculi & 302 & $159(77.18)$ & $143(69.42)$ & 0.075 \\
\hline AP & 57 & $27(13.11)$ & $30(14.56)$ & 0.669 \\
\hline Suppurative cholangitis & 70 & $27(13.11)$ & $43(20.87)$ & 0.036 \\
\hline Cholangiocarcinoma & 52 & $32(15.53)$ & $20(9.71)$ & 0.063 \\
\hline Papillary carcinoma of duodenum & 9 & $2(0.97)$ & $7(3.40)$ & 0.178 \\
\hline Duodenal adenomas & 6 & $1(0.49)$ & $5(2.43)$ & 0.217 \\
\hline Pancreatic head cancer & 9 & $2(0.97)$ & $7(3.40)$ & 0.178 \\
\hline Biliary stent removal & 12 & $4(1.94)$ & $8(3.88)$ & 0.241 \\
\hline Chronic pancreatitis & 3 & $3(1.46)$ & $0(0)$ & 0.246 \\
\hline Biliary ascariasis & 0 & $0(0)$ & $0(0)$ & - \\
\hline Unknown aetiology of CBD dilatation & 10 & $3(1.46)$ & $7(3.40)$ & 0.200 \\
\hline
\end{tabular}

$P A D$ - periampullary diverticulum, $A P$ - acute pancreatitis, $C B D$ - common bile duct. *Statistically significant.

of the grading of bleeding, moderate and severe bleeding did not differ between groups, except for mild ones $(p=0.043)$. The percentage of failed cannulation did not significantly differ between groups $(1.02 \%$ in PAD vs. $1.03 \%$ in non-PAD; $p>0.05)$. Although the incidence of gastrointestinal perforation was rarely seen, a significant difference was observed in the perforation rate be- tween the two groups $(1.02 \%$ in PAD vs. $0.09 \%$ in non-PAD; $p=0.028)$. Additionally, developments of 24 cases of PEP (8.19\%) and 35 cases of hyperamylasaemia (11.95\%) were observed in the PAD group; whereas, 30 (2.58\%) cases and 81 (6.97\%) cases of PEP and hyperamylasaemia, respectively, were detected in the non-PAD group. The difference was statistically significant $(p<0.05)$. 
To adjust for differences in baseline clinical characteristics according to PAD status, a $1: 1$ propensity score matching was performed, as shown in Table V. All clinical variables that can affect post-ERCP complications were adjusted and compared after propensity score matching. Gastrointestinal bleeding was significantly higher in patients with PAD compared to the non-PAD group ( $8.25 \%$ vs. $3.40 \%$, $p=0.035)$ but did not associate with the grading of it, after propensity score matching. Both before and after propensity score matching, PEP and hyperamylasaemia occurred observably more frequently in patients with PAD. While the perforation rates were significantly higher in the PAD group, there was no statistical difference after propensity score matching (Table IV).

\section{Discussion}

Consistent with previous publications, our study showed equal frequency of occurrence of PAD in both genders. However, the prevalence and detection rate of PAD have a strong association with age [13]. This is partially due to the fact that PAD is rarely seen in subjects under 40 years old, whereas it increases swiftly from the age of 50 years [9]. In this study, the PAD group had higher mean age than the control group, with more than $60 \%$ of the PAD cases detected in older patients ( $\geq 60$ years), illustrating that PAD occurred more often in the elderly. A possible explanation might be that diverticula can be attributable to intestinal smooth muscle dysfunction resulting from vagal attrition and a degenerative process involving local supporting structures associated with aging [14-16]. An additional explanation might be that under increased intraduodenal pressure conditions, an outpouching of the intestinal wall at its weakest points, for example, regions surrounding the papilla duodeni major (opening of the pancreatic duct into the duodenum) could be possible.

Clinically, PAD has been shown to be associated with biliary diseases because it is believed to contribute to biliary calculi formation [17], especially CBD stones [18]. In the present study, we noticed a higher incidence of biliary calculi in PAD patients (81.91\%) compared to non-PAD patients (74.96\%), which is consistent with previous observations. Recent studies showed that biliary calculi and cholangitis tend to occur more often in subjects with PAD, and these studies considered its presence as an independent risk factor for CBD stones secondary to acute cholangitis. This is probably due to a mechanical compression of the CBD, which in turn increases the pressure of the bile duct, resulting in disturbances of bile excretion [19]. Additionally, accumulation of food in the PAD with a secondary bacterial infection could lead to chronic fibrosis or stricture of the sphincter of Oddi, ultimately contributing to pancreatobiliary reflux, biliary bacterial infection, the formation of cholangitis, and bile duct stones [8, 20, 21]. As shown in our study, we found a clear association between diverticula and a higher incidence of biliary calculi and primary choledocholithiasis. Interestingly, cholecystolithiasis with CBD stones was more common in the non-PAD group than in the PAD group. The specific mechanism remains unclear. We also showed that the PAD type had no effect on the formation of bile duct stones and that, consistent with previous reports made by Ozogul et al. [8] and Kim et al. [10], giant diverticulum and suppurative cholangitis occurred more frequently in type I and III, respectively. Furthermore, similar to previous works, our study indicated that patients in the PAD group had a higher prevalence of primary CBD stones and suppurative cholangitis compared to their counterparts $[22,23]$. Interestingly, the PAD group were prone to complications due to multiple small stones $(D<1 \mathrm{~cm}, N>2)$. This finding led us to think that small stones may play a role in the obstruction of the opening of the duodenal papilla, which in turn might explain the predisposition of patients with PAD to suppurative cholangitis.

Zoepf et al. suggested that AP probably derived from the formation of bile duct stones rather than direct association with PAD [24]. Nevertheless, other theories argued that PAD could directly contribute to the occurrence of AP and might even be considered as an independent risk factor in elderly patients [25]. It is possible that distension of PAD with inspissated food or giant diverticulum might play a role in pancreatic duct compression. Another possibility might be related to the position of PAD in relation to the opening of the papilla, which, if too close, may put pressure on the terminal parts of the bile duct and interfere with pancreatic juice drainage, thus resulting in pancreatitis. Our study further categorised and compared the aetiology of AP in both groups. The result showed that the occurrence of an association between AP and biliary calculi, cholangiocarcinoma, or carcinoma of the pancreatic head was more common in the PAD group. Additional analysis determined no correlation between PAD type and AP occurrence. However, whether AP is directly caused by PAD or by other indirect factors is still debatable because initial clinical data indicated the presence of pancreaticobiliary diseases in several patients. Subsequently, we theorised that PAD might play a role in the incidence of biliary pancreatitis and idiopathic pancreatitis.

Due to the high occurrence rate of acute biliary pancreatitis, AP, and recurrent pancreatitis in PAD patients [25], many theories have suggested that PAD might have a more intimate association with 
chronic pancreatitis. In our study, even though no statistical significance $(p>0.05)$ was observed, we can still see that the frequency of chronic pancreatitis was higher in the PAD group compared to the control group. The lack of significant difference between groups in our study might be due to the smaller number of chronic pancreatitis cases, and the issue remains to be further investigated with larger sample sizes [26]. We also found that carcinoma of the pancreatic head was more frequent in the PAD group compared to their counterparts. This is probably due to complications induced by the presence of biliary stones or biliary infection, which in turn increase the risk of AP or chronic pancreatitis resulting in injured pancreatic acinar cells and dysplasia. However, the exact pathogenesis remains to be further investigated.

Previously, PAD was considered as one of the main reasons for ERCP failure. Its presence is thought to make ERCP a technically challenging procedure with significantly lower success rate $[2,5,6,9,13]$. Periampullary diverticulum was often associated with prolonged procedure $[2,5]$ and a higher cannulation failure [27]. However, more recent studies have shown that a low cannulation rate can be attributed to the inability of endoscopists to detect abnormal or allotropic papilla in a substantial percentage of cases with giant PAD. Meanwhile, the presence of PAD had no significant impact on the success rate of ERCP $[1,5,28]$. Contrarily, some studies demonstrated that the presence of PAD during ERCP could even be considered as an independent predictor of more convenient cannulation $[15,29]$. The failure rate of ERCP between the two groups did not differ significantly in this study $(1.02 \%$ vs. $1.03 \% ; p>0.05)$.

Complications during ERCP, as an invasive procedure, are probably inevitable, but whether PAD is only a benign bystander or a major contributor remains unclear. According to a previous study, incidences of hyperamylasaemia and PEP were observed, respectively, in $7.7 \sim 19.7 \%$ and about 5\% of cases [30]. In our study, the occurrence rates of hyperamylasaemia and PEP were significantly higher in the PAD group, similar to findings made by Parlak et al. [31]. However, no significant association between PAD type and incidences of hyperamylasaemia and PEP was observed. This shows that even though PAD may be responsible for the increased frequency of hyperamylasaemia and PEP, current findings are still insufficient to point out the relationship, if any, between PAD type and their occurrence. Another cause of ERCP-related complications is probably oedema of the papilla due to repeated cannulation, which can lead to difficulties of drainage of pancreatic juice. The commonly proposed solution to miti- gate this problem is the usage of pancreatic duct guidewire-indwelling, which decreases the risk of repeated intubation but, conversely, increases the incidence of hyperamylasaemia or PEP.

Periampullary diverticulum may also provide additional risks for the development of bleeding [24], the most common ERCP complication, due to the following factors: a) The technical difficulty in attempting successful cannulation in patients with PAD is higher, especially when the papilla cannot be located. The need for an additional EPBD, EST, and repeated cannulation will inevitably increase the risk of bleeding. b) It is possible for PAD to cause deformation of adjacent vessels due to their abundance in the papilla of the duodenum and the lower end of CBD. This increases the risk of damage during EST. Results from the present study indicated that bleeding develops more frequently in the PAD group and that PAD may represent an additional risk to the development of bleeding.

The incidence of perforation secondary to ERCP ranges from $0.5 \%$ to $1.8 \%$ in China, and $0.3 \%$ to $0.6 \%$ in foreign countries. Perforations are mainly located around the papilla or the descending part of the duodenum. Several published observations underscored the significant role that PAD can play in the incidence of ERCP-related gastrointestinal perforation. It is possible that the structural composition of the diverticulum wall is only comprised of a mucosa layer and a serous membrane layer, which makes it weaker than a normal one [32]. Interestingly, results from our study conversely show no obvious association between PAD and perforation, which might result from the smaller number of perforation cases.

Although more studies are still needed, our findings indicate that PAD is closely related to the occurrence of various pancreaticobiliary diseases and suggest that efforts to monitor the presence of PAD might be more beneficial than previously thought. Our study focused on analysing the association of PAD with pancreaticobiliary diseases and the risk as well as adverse events of the ERCP procedure, subsequently providing necessary clinical experience and information to the endoscopists. The main limitation of this study is its retrospective and single-centred nature, which limits the extrapolation of its data to other centres.

In conclusion, although the incidence of PAD may be an indicator of increased occurrence of pancreaticobiliary diseases and ERCP-related complications, except for perforation, and is positively correlated with age, its finding during ERCP should not be considered as an obstacle to a successful cannulation, which, on the contrary, is more dependent on our proficiency in endoscopic manipulation. 


\section{Acknowledgments}

We are grateful to all the participants and colleagues for their contributions.

\section{Conflict of interest}

The authors declare no conflict of interest.

\section{References}

1. Sun Z, Bo W, Jiang P, Sun Q. Different types of periampullary duodenal diverticula are associated with occurrence and recurrence of bile duct stones: a case-control study from a Chinese center. Gastroenterol Res Pract 2016; 2016: 9381759.

2. Altonbary AY, Bahgat MH. Endoscopic retrograde cholangiopancreatography in periampullary diverticulum: the challenge of cannulation. World J Gastrointest Endosc 2016; 8: 282-7.

3. Osipenko MF, Bikbulatova EA. Colon diverticula: origin, prevalence, clinical manifestations. Ter Arkh 2007; 79: 26-31.

4. Katsinelos P, Chatzimavroudis G, Tziomalos K, et al. Impact of periampullary diverticula on the outcome and fluoroscopy time in endoscopic retrograde cholangiopancreatography. Hepatobiliary Pancreat Dis Int 2013; 12: 408-14.

5. Coman IS, Radu EV, Sandru V, et al. Retained common bile duct lithiasis at a patient with periampullary duodenal diverticulum. Chirurgia (Bucur) 2016; 111: 517-21.

6. Kang HS, Hyun JJ, Kim SY, et al. Lemmel's syndrome, an unusual cause of abdominal pain and jaundice by impacted intradiverticular enterolith: case report. J Korean Med Sci 2014; 29: 874-8.

7. Kim KH, Kim TN. Endoscopic papillary large balloon dilation in patients with periampullary diverticula. World J Gastroenterol 2013; 19: 7168-76.

8. Ozogul B, Ozturk G, Kisaoglu A, Aydinli B, Yildirgan M, Atamanalp SS. The clinical importance of different localizations of the papilla associated with juxtapapillary duodenal diverticula. Can J Surg 2014; 57: 337-41.

9. Zippi M, Traversa G, Pica R, et al. Efficacy and safety of endoscopic retrograde cholangiopancreatography (ERCP) performed in patients with Periampullary duodenal diverticula (PAD). Clin Ter 2014; 165: e291-4.

10. Kim CW, Chang JH, Kim JH, Kim TH, Lee IS, Han SW. Size and type of periampullary duodenal diverticula are associated with bile duct diameter and recurrence of bile duct stones. J Gastroenterol Hepatol 2013; 28: 893-8.

11. Cotton PB, Lehman G, Vennes J, et al. Endoscopic sphincterotomy complications and their management: an attempt at consensus. Gastrointest Endosc 1991; 37: 383-93.

12. Christoforidis E, Goulimaris I, Kanellos I, Tsalis K, Demetriades C, Betsis D. Post-ERCP pancreatitis and hyperamylasemia: patient-related and operative risk factors. Endoscopy 2002; 34: 286-92.

13. Mohammad AA, Afzali ES, Shahnazi A, et al. ERCP features and outcome in patients with periampullary duodenal diverticulum. ISRN Gastroenterol 2013; 2013: 217261.

14. Boix J, Lorenzo-Zuniga V, Ananos F, Domenech E, Morillas RM, Gassull MA. Impact of periampullary duodenal diverticula at endoscopic retrograde cholangiopancreatography: a proposed classification of periampullary duodenal diverticula. Surg Laparosc Endosc Percutan Tech 2006; 16: 208-11.

15. Tyagi P, Sharma P, Sharma BC, Puri AS. Periampullary diverticula and technical success of endoscopic retro- grade cholangiopancreatography. Surg Endosc 2009; 23: $1342-5$

16. Yun AJ, Bazar KA, Lee PY. A new mechanism for diverticular diseases: aging-related vagal withdrawal. Med Hypotheses 2005; 64: 252-5.

17. Lee JJ, Brahm G, Bruni SG, Thipphavong S, Sreeharsha B. Biliary dilatation in the presence of a periampullary duodenal diverticulum. Br J Radiol 2015; 88: 20150149.

18. Major P, Dembinski M, Winiarski $M$, et al. A periampullary duodenal diverticula in patient with choledocholithiasis - single endoscopic center experience. Pol Przegl Chir 2016; 88: 328-33.

19. Miyazaki S, Sakamoto T, Miyata M, Yamasaki Y, Yamasaki H, Kuwata K. Function of the sphincter of Oddi in patients with juxtapapillary duodenal diverticula: evaluation by intraoperative biliary manometry under a duodenal pressure load. World J Surg 1995; 19: 307-12.

20. Egawa N, Anjiki H, Takuma K, Kamisawa T. Juxtapapillary duodenal diverticula and pancreatobiliary disease. Dig Surg 2010; 27: 105-9.

21. van Nieuwkoop C, Boere I, Rosekrans PA, Bac DJ. Recurrent bacterial cholangitis due to a juxtapapillary diverticulum. Eur J Gastroenterol Hepatol 2002; 14: 189-90.

22. Bruno M, Brizzi RF, Mezzabotta L, et al. Unexplained common bile duct dilatation with normal serum liver enzymes: diagnostic yield of endoscopic ultrasound and follow-up of this condition. J Clin Gastroenterol 2014; 48: e67-70.

23. Tomizawa M, Shinozaki F, Motoyoshi Y, Sugiyama T, Yamamoto S, Sueishi M. Association between juxtapapillary diverticulum and acute cholangitis determined using laboratory data. Clin Exp Gastroenterol 2014; 7: 447-51.

24. Zoepf T, Zoepf DS, Arnold JC, Benz C, Riemann JF. The relationship between juxtapapillary duodenal diverticula and disorders of the biliopancreatic system: analysis of 350 patients. Gastrointest Endosc 2001; 54: 56-61.

25. Uomo G, Manes G, Ragozzino A, Cavallera A, Rabitti PG. Periampullary extraluminal duodenal diverticula and acute pancreatitis: an underestimated etiological association. Am J Gastroenterol 1996; 91: 1186-8.

26. Naranjo-Chavez J, Schwarz M, Leder G, Beger HG. Ampullary but not periampullary duodenal diverticula are an etiologic factor for chronic pancreatitis. Dig Surg 2000; 17: 358-63.

27. Loffeld RJ, Dekkers PE. The impact of duodenal diverticuli and the execution of endoscopic retrograde cholangiopancreaticography. Int Sch Res Notices 2016; 2016: 5026289.

28. Chen L, Xia L, Lu Y, Bie L, Gong B. Influence of periampullary diverticulum on the occurrence of pancreaticobiliary diseases and outcomes of endoscopic retrograde cholangiopancreatography. Eur J Gastroenterol Hepatol 2017; 29: 105-11.

29. Panteris V, Vezakis A, Filippou G, Filippou D, Karamanolis D, Rizos S. Influence of juxtapapillary diverticula on the success or difficulty of cannulation and complication rate. Gastrointest Endosc 2008; 68: 903-10.

30. Elmunzer BJ, Scheiman JM, Lehman GA, et al. A randomized trial of rectal indomethacin to prevent post-ERCP pancreatitis. N Engl J Med 2012; 366: 1414-22.

31. Parlak E, Suna N, Kuzu UB, et al. Diverticulum with papillae: does position of papilla affect technical success? Surg Laparosc Endosc Percutan Tech 2015; 25: 395-8.

32. Takano Y, Nagahama M, Yamamura E, Maruoka N, Takahashi $\mathrm{H}$. Perforation of the papilla of vater in wire-guided cannulation. Can J Gastroenterol Hepatol 2016; 2016: 5825230 . 